\title{
Secure Messaging, Diabetes Self-management, and the Importance of Patient Autonomy: a Mixed Methods Study
}

\author{
Stephanie A. Robinson, $P h D^{1,2}$, Mark S. Zocchi, MPH ${ }^{1,3}$, Dane Netherton, $P h D^{1,4}$, \\ Arlene Ash, $\mathrm{PhD}^{4}$, Carolyn M. Purington, $\mathrm{MPH}^{7}$, Samantha L. Connolly, PhD ${ }^{5,6}$, \\ Varsha G. Vimalananda, MD, MPH ${ }^{1,7}$, Timothy $P$. Hogan, $P h D^{1,8}$, and \\ Stephanie L. Shimada, PhD ${ }^{1,4,9}$
}

\begin{abstract}
${ }^{1}$ Center for Healthcare Organization and Implementation Research, Edith Nourse Rogers Memorial Veterans Hospital, Bedford, MA, USA; ${ }^{2}$ Boston University School of Medicine, Boston, MA, USA; ${ }^{3}$ The Heller School for Social Policy and Management, Brandeis University, Waltham, MA, USA; ${ }^{4}$ University of Massachusetts Medical School, Worcester, MA, USA; ${ }^{5}$ Center for Healthcare Organization and Implementation Research, VA Boston Healthcare System, Boston, MA, USA; ${ }^{6}$ Department of Psychiatry, Harvard Medical School, Boston, MA, USA; ${ }^{7}$ School of Medicine, Boston University, Boston, MA, USA; ${ }^{8}$ Department of Population and Data Sciences, University of Texas Southwestern Medical Center, Dallas, TX, USA; ' 5 School of Public Health, Boston University, Boston, MA, USA
\end{abstract}

BACKGROUND: Diabetes is a complex, chronic disease that requires patients' effective self-management between clinical visits; this in turn relies on patient self-efficacy. The support of patient autonomy from healthcare providers is associated with better self-management and greater diabetes self-efficacy. Effective provider-patient secure messaging (SM) through patient portals may improve disease self-management and self-efficacy. SM that supports patients' sense of autonomy may mediate this effect by providing patients ready access to their health information and better communication with their clinical teams.

OBJECTIVE: We examined the association between healthcare team-initiated SM and diabetes selfmanagement and self-efficacy, and whether this association was mediated by patients' perceptions of autonomy support from their healthcare teams.

DESIGN: We surveyed and analyzed content of messages sent to a sample of patients living with diabetes who use the SM feature on the VA's My HealtheVet patient portal.

PARTICIPANTS: Four hundred forty-six veterans with type 2 diabetes who were sustained users of SM.

MAIN MEASURES: Proactive (healthcare team-initiated) SM (0 or $\geq 1$ messages); perceived autonomy support; diabetes self-management; diabetes self-efficacy.

KEY RESULTS: Patients who received at least one proactive SM from their clinical team were significantly more likely to engage in better diabetes self-management and report a higher sense of diabetes self-efficacy. This relationship was mediated by the patient's perception of autonomy support. The majority of proactive SM discussed scheduling, referrals, or other administrative content. Patients' responses to team-initiated communication promoted patient engagement in diabetes self-management behaviors. CONCLUSIONS: Perceived autonomy support is important for diabetes self-management and self-efficacy. Proactive communication from clinical teams to patients can

Received September 2, 2019

Accepted April 1, 2020

Published online May 21, 2020 help to foster a patient's sense of autonomy and encourage better diabetes self-management and self-efficacy.

KEY WORDS: patient portal; veterans; patient-provider communication; diabetes; qualitative; mediation; patient autonomy.

J Gen Intern Med 35(10):2955-62 DOI: $10.1007 / \mathrm{s} 11606-020-05834-\mathrm{x}$

(c) Society of General Internal Medicine (This is a U.S. government work and not under copyright protection in the U.S.; foreign copyright protection may apply) 2020

\section{INTRODUCTION}

In a complex chronic disease such as diabetes, between-visit communication among patients and healthcare personnel can play a critical role in supporting patients' self-efficacy and disease self-management. Patient portals can support betweenvisit communication by improving patients' access to their health information and enable better communication with their clinical teams. ${ }^{1,2}$ Most patient portals offer patients the opportunity to communicate with their clinical teams via asynchronous secure messaging (SM). Previous studies have found that patients' use of SM was associated with improvements in their diabetes care and outcomes such as hemoglobin A1c (HbAlc). ${ }^{3-}$ ${ }^{5}$ However, SM is often underutilized by patients, suggesting a potential missed opportunity to support diabetes management. ${ }^{6}$ Recent work has demonstrated that provider-initiated, proactive $\mathrm{SM}$ is associated with higher rates of patient $\mathrm{SM}^{7}$. Therefore, provider-initiated SM may be a critical component to effectively encouraging diabetes self-management and self-efficacy.

Self-determination theory incorporates three important constructs: autonomy support, self-management, and self-efficacy. ${ }^{8}$ Autonomy support, the central concept in this theory, specifically refers to patients' perceptions that their healthcare providers support their self-care choices and actions. An autonomy-supportive environment increases intrinsic 
motivation for sustained health self-management behaviors, 8,9 such as improved medication adherence, ${ }^{10}$ greater diabetes selfefficacy, ${ }^{11}$ and good glycemic control among patients with type 2 diabetes. ${ }^{10}$ Previous work demonstrated improvements in glycemic control ${ }^{12}$ and diabetes distress ${ }^{13}$ among patients with type 2 diabetes which in turn was associated with autonomous motivation and an autonomy-supportive healthcare climate. ${ }^{12}$ Healthcare professionals can support patients' sense of autonomy by acknowledging their perspectives, providing meaningful rationales for self-management behaviors, offering and supporting patient participation in health-related decisions, and fostering internal sources of motivation. ${ }^{13,14}$

This study extends previous research by examining the influence of healthcare team-initiated SM behavior on patients' perceived autonomy support, diabetes self-management, and self-efficacy. We hypothesized that patients who received team-initiated SM would report their healthcare as more autonomy-supportive compared to patients that received no team-initiated SM. We also hypothesized that patients who see their healthcare teams as more autonomysupportive would report greater engagement in diabetes selfmanagement and greater diabetes self-efficacy. Finally, we hypothesized that the relationship between team-initiated SM and diabetes self-management and self-efficacy could be mediated by the patients' perception of autonomy support. Figure 1 represents our hypothesized conceptual model.]->

\section{METHODS}

\section{Recruitment}

The Edith Nourse Rogers Memorial Veterans Hospital's Institutional Review Board approved this study. This analysis used data collected in a larger study examining the role of patient portal use in diabetes management. In that study, we identified 3802 veterans with type- 2 diabetes who had uncontrolled blood glucose (2012 mean A1C > 8.0\% and less than $25 \%$ of the year with an $\mathrm{A} 1 \mathrm{C}<8.0 \%$ ), who were sustained users of My Health$\boldsymbol{e}$ Vet, the VA's patient portal. As a single instance of portal use could reflect patient efforts to explore or test a feature, a sustained user was defined according to repeated and current use of key portal features. Repeated use included having requested prescription refills 2 or more times, viewed or downloaded their health information 2 or more times, and used SM 2 or more

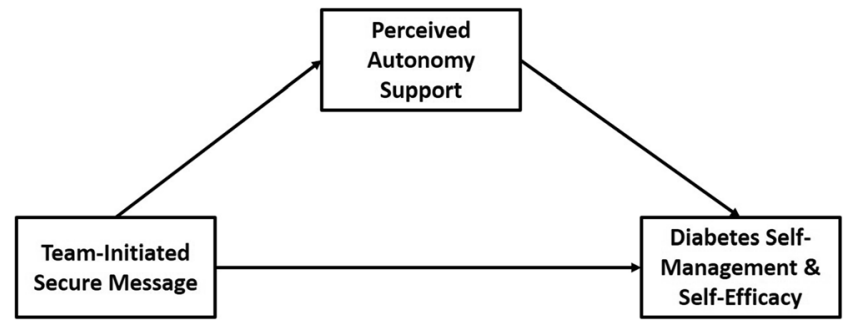

Figure 1 Conceptual model of the indirect effect of patient autonomy support on the relationship between team-initiated SM and diabetes self-management and self-efficacy. times in 2 out of 3 years between 2013 and 2015. Current use included having sent at least four SM between January 2016 and June 2017. To identify a sample with diverse starting A1C levels, we divided this group into those who achieved A1C control by $2016(n=1474)$ and those who remained uncontrolled in $2016(n=2328)$. Starting with those who achieved control, we used stratified random sampling to select a subset of veterans balanced on location (rural vs. urban) and mental health status (comorbid mental health diagnoses vs. none) from each of the four strata (rurality $\times$ mental health). We then selected patients who remained uncontrolled, matched in terms of age and mean A1C in 2012. We mailed surveys to 1000 veterans in November 2017. Figure 2 depicts our sampling method. To achieve an optimal response rate, 200 additional surveys (100 achieved control, and 100 remained uncontrolled) were mailed at the beginning of 2018.] $\rightarrow$

\section{Measures}

Team-Initiated Secure Messages. We examined survey respondents' 2017 SM threads. Each SM was coded individually to indicate whether it was proactively initiated by the respondent's healthcare team (i.e., the first SM was sent by a healthcare team member). Each message was summarized and coded as containing either no (0) or any $(\geq 1)$ team member-initiated messages.

Outcome Measures. Perceived Autonomy Support. The Health Care Climate Questionnaire $\left(\mathrm{HCCQ}^{15}\right)$ measured perceived autonomy support. This 6-item survey measures patients' perceptions of the degree to which their healthcare provider supports their autonomy as a patient (e.g., "my provider provides me choices and options," "my provider listens to how I would like to do things"). Each item was on a scale from 1 (strongly disagree) to 7 (strongly agree). A composite score was formed by averaging across items.

Diabetes Self-management. Diabetes self-management behaviors were measured with the Diabetes SelfManagement Questionnaire (DSMQ $\left.{ }^{16}\right)$. The DSMQ is a global measure of diabetes self-management comprised of 16 items to assess activities related to glycemic control in patients with diabetes (e.g., "I check my blood sugar levels with care and attention"; "I keep all of my doctors' appointments recommended for my diabetes treatment"). It asks participants to rate how much they apply to them on a scale from 0 (does not apply to me) to 3 (applies to me very much). A composite score was calculated as the average of four subscales: glucose management, dietary control, physical activity, and healthcare use, and could range from 0 to 10 . Higher values indicate more effective self-management. The DSMQ has been shown to be significantly correlated with A1C levels. ${ }^{16}$

Diabetes Self-efficacy. Diabetes self-efficacy was measured with the Diabetes Self-efficacy Scale (DSES ${ }^{17}$ ). Respondents 


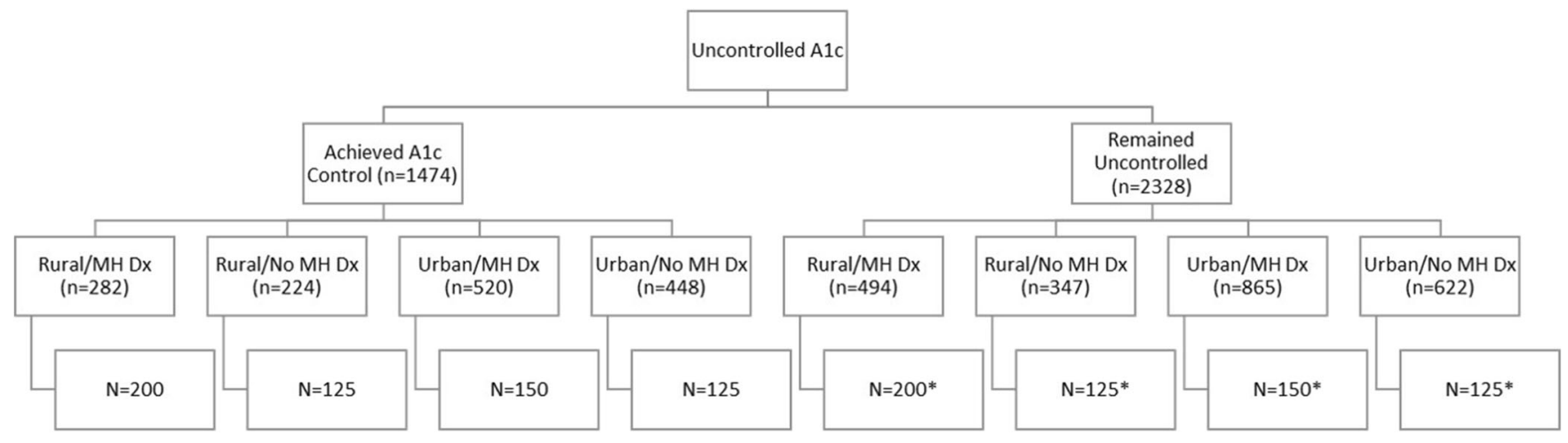

Figure 2 Survey sampling schema. MH Dx = comorbid mental health diagnosis; No MH= no comorbid mental health diagnosis. *Sample was matched on age, gender, etc. to their "Achieved A1c Control" counterparts.

indicated from 1 (not at all confident) to 10 (totally confident) how confident they are in their ability to do certain activities related to managing their diabetes (e.g., "knowing what to do when blood sugar level goes higher or lower than it should be," or "controlling diabetes so that it does not interfere with things you want to do"). The score for the scale is the mean of the eight items and can range from 1 to 10 .

Analysis. Relying on survey, health record information, and SM coding, we used a mixed methods design (QUAN $\rightarrow$ qual $)^{18}$ to quantify the relationship between clinical teaminitiated SM and patient-reported measures (perceived autonomy, diabetes self-management, and diabetes self-efficacy) and examine the qualitative content. Data were analyzed using Hayes' PROCESS model in the SAS Enterprise Guide. ${ }^{19}$ Two separate models were run. One estimated the direct and indirect effect of team-initiated SM and diabetes self-management through perceived autonomy support. The other estimated the direct and indirect effect of team-initiated SM and diabetes self-efficacy through perceived autonomy support. Covariates included age (continuous), gender (male or female), and income. Income was self-reported on a 16-item scale ranging from "less than $\$ 5,000$ " to "more than $\$ 150,000$." We dichotomized income as less than $\$ 35,000$ (46.4\% of the sample) and $\$ 35,000$ or above $(53.6 \%)$. These covariates were included in all models.

SMs within team-initiated threads were coded for (a) content and (b) sender (e.g., patient, physician, registered nurse, pharmacist) based on examining the signature block that accompanied a SM. Each SM was read and categorized based on previously published message coding methods. ${ }^{20-23} \mathrm{We}$ coded it with binary indicators for whether the SMs were related to each of the following topics: scheduling, referrals, or administrative tasks; medication renewals or refills; other medication-related issues; test results; test issues; health issues; self-reporting; informational; technology-related; life issues; complaints; establishing a personal connection; care coordination; unknown/other. Each message could be coded for more than one topic (e.g., refills and scheduling). All messages were double-coded by two of three trained research assistants who met regularly to discuss questions, reach agreement on all coding discrepancies, and refine the coding categories.

\section{RESULTS}

Out of the 1200 surveys sent, 446 patients responded (response rate $=37.2 \%$ ). We compared responders to nonresponders (Table 1). Overall, responders were slightly older $(p=0.032)$ and less likely to have a mental health diagnosis $(p<0.001)$ compared to non-responders. Responders logged onto My HealtheVet more frequently than non-responders $(p=0.012)$ and refilled more prescriptions through the portal $(p=0.041)$.

A large majority $(75.4 \%, n=331)$ of responders never received a proactively initiated SM from their healthcare teams, although 108 (24.6\%) did. Among those that received a team-initiated SM, the median number of SMs received was two. Overall perceptions of their team's autonomy support was relatively high (mean $=5.16, \mathrm{SD}=1.86$, range $=1-7$ ), as was their reported engagement in diabetes self-management ( mean $=7.88, \mathrm{SD}=0.89$, range $=5.00-9.84$ ), and degree of diabetes self-efficacy, reflecting their confidence in being able to manage their diabetes (mean $=7.20, \mathrm{SD}=1.90$, range $=1-$ 10). Table 2 shows correlations among the study variables.

\section{Diabetes Self-management}

Patients who received $\geq 1$ team-initiated SM from their clinical team reported more autonomy support than patients who received $0(B=0.43, \mathrm{SE}=0.21, p=0.046)$. The relationship between perceived autonomy support and diabetes selfmanagement persisted after adjusting for covariates and team-initiated $\mathrm{SM}(B=0.09, \mathrm{SE}=0.02, p<0.001)$. Adjusting for age, gender, and income, there was no significant difference (i.e., direct effect) in reported diabetes self-management behaviors between patients that received a team-initiated SM and patients that did not. However, there was a significant indirect effect; patients that received at least one team-initiated 
Table 1 Sample Characteristics

\begin{tabular}{|c|c|c|c|}
\hline & Responders & No response & $p$ value \\
\hline$N$ & 446 & 754 & - \\
\hline Age, mean (SD) & $66.4(7.5)$ & $65.3(8.6)$ & 0.0315 \\
\hline \multicolumn{4}{|l|}{ Gender, $N(\%)$} \\
\hline Male & $418(93.7)$ & $710(94.2)$ & 0.755 \\
\hline \multicolumn{4}{|l|}{ Race, $N(\%)$} \\
\hline White & $384(86.1)$ & $628(83.3)$ & 0.421 \\
\hline African-American or Black & $39(8.7)$ & $84(11.1)$ & \\
\hline Asian & $0(0)$ & $3(0.4)$ & \\
\hline American Indian or Alaska Native & $1(0.2)$ & $5(0.7)$ & \\
\hline Native Hawaiian or other Pacific Islander & $6(1.3)$ & $11(1.5)$ & \\
\hline Other/missing & $16(3.6)$ & $23(3.1)$ & \\
\hline \multicolumn{4}{|l|}{ Income, $N(\%)$} \\
\hline$<\$ 35,000$ & $207(46.4 \%)$ & - & \\
\hline \multicolumn{4}{|l|}{ Rurality, $N(\%)$} \\
\hline Rural & $228(51.1)$ & $422(56.0)$ & 0.103 \\
\hline \multicolumn{4}{|l|}{ Mental health diagnosis, $N(\%)$} \\
\hline With diagnosis & $233(52.2)$ & $472(62.6)$ & $<0.001$ \\
\hline A1c\% TIC 2017, mean (SD) & $49.6(43.4)$ & $49.3(43.8)$ & 0.9096 \\
\hline \multicolumn{4}{|l|}{ A1c control status, $N(\%)$} \\
\hline Remained uncontrolled in 2016 & $225(50.5)$ & $375(49.7)$ & 0.811 \\
\hline \multicolumn{4}{|l|}{ MHV Utilization 2017, median (IQR) } \\
\hline Days using MHV (any activity) & $50(33-73)$ & $44(26-65)$ & 0.012 \\
\hline Days using secure messaging & $11(6-20)$ & $10(5-17)$ & 0.086 \\
\hline Days using prescription refills & $14(9-22)$ & $13(8-19)$ & 0.041 \\
\hline Days using blue button & $1(0-3)$ & $1(0-3)$ & 0.357 \\
\hline Days viewing appointments & $13(6-26)$ & $12(5-24)$ & 0.186 \\
\hline \multicolumn{4}{|l|}{ Health Care Use 2017, mean (SD) } \\
\hline Primary care visits & $10.0(7.7)$ & $9.7(7.6)$ & 0.510 \\
\hline \multicolumn{4}{|l|}{ Team-initiated secure message, $N(\%)$} \\
\hline None & $331(74.2)$ & 538 (71.4) & 0.284 \\
\hline
\end{tabular}

Alc\%TIC = percent of time spent in control $($ Alc $<8 \%) ; M H V=$ My HealtheVet

SM reported better diabetes self-management via their higher perceived autonomy support $(B=0.04,95 \% \mathrm{CI}=0.003$, 0.082; Table 3).

\section{Diabetes Self-efficacy}

There was a marginally significant difference in perceived autonomy support between patients who received $\geq 1$ SM compared to patients who received 0 team-initiated SMs $(B=0.39, \mathrm{SE}=0.21, p=0.065)$. Adjusting for covariates and team-initiated SM, there was a significant relationship between perceived autonomy support and diabetes self-efficacy $(B=0.25, \mathrm{SE}=0.05, p<0.001)$. There was no significant direct effect between receiving a team-initiated SM and diabetes self-efficacy. The indirect effect between team-initiated SM and diabetes self-efficacy through perceived autonomy support was marginally significant $(B=0.10,95 \% \mathrm{CI}=-$ $0.003,0.214)$.

\section{Team-Initiated Secure Message Content}

For a deeper understanding of the relationships we identified, we examined the content of the team-initiated SM and patient responses (Table 4). The majority of team-initiated SM were sent by a registered nurse $(41 \%, n=93)$. SM sent by physicians $(13 \%, n=29)$ and SM in which the sender was not clearly identified $(13 \%, n=29)$ were the next most frequent initiators of SMs.
Overall, the most common topic in team-initiated messages related to scheduling, referrals, or administrative content $(n=$ $83,36 \%$ ). Many of these SMs were straightforward requests to schedule or reschedule appointments. For example, in the following quote, a medical assistant attempted to schedule lab work on behalf of a patient: "You are due for your A1c labs this month. What day can I get you scheduled." A common theme related to scheduling that emerged was the use of SM to reach out to patients whom the team was not able to contact via phone.

...We have tried to contact you by telephone to schedule an appointment; but so far have not been able to reach you. We are sending this notification via secure messaging in hopes of scheduling your appointment... If we do not hear from you we will assume you do not wish to obtain this care and will return the consult to your clinical provider. - Other VA team member

The second most commonly discussed topic in teaminitiated messages were medication issues $(n=72 ; 31 \%)$. For example, one nurse informed the patient of possible adverse consequences to a current medication.

...It is recommended that your blood pressure medication (lisinopril) be replaced. Do you agree to having the Iisinopril replaced? It is very important that you stop 
Table 2 Correlations

\begin{tabular}{lllll}
\hline \hline & Age & Team-initiated SM & perceived autonomy & Self-management \\
\hline Age & - & & & \\
Team-initiated SM & 0.02 & - & & \\
Perceived autonomy & 0.08 & $0.10^{*}$ & - & $-19^{* * *}$ \\
Self-management & $0.15^{* *}$ & -0.01 & $0.25^{* * *}$ & $0.54^{* * *}$ \\
Self-efficacy & 0.07 & $-0.12^{*}$ & & - \\
\hline
\end{tabular}

SM= secure message

$* p<.05, * * p<0.01, * * * p<0.001$

the use of any non-steroids anti-inflammatory drug (ie: Motrin, Ibuprofen, Aleve, Goody powder) and that you stay well hydrated (5-6 cups of fluids/water in a day). VA Registered Nurse

The nurse explained what the medication issue was and asked if the patient agreed to have it replaced. The nurse also recommended some precautions (e.g., stay hydrated and avoid anti-inflammatory medication).

The third most common topics were test results $(n=15$, $19 \%)$ and medication renewals $(n=15 ; 19 \%)$. Test results were commonly sent to the patient via SM. For example, a registered nurse and diabetes educator informed the patient about an improvement in A1c and attached the lab results to allow the patient to compare these results to other results: "Your A1c improved. Here are lab results so you can compare!" Clinical team members often used SM to communicate about medication renewals, as well.

... As you requested, a prescription for Aspirin 81mg daily is being mailed for you to take one tablet daily... begin taking the 10 units of insulin immediately before you eat rather than afterwards... I would like to begin monitoring your blood sugars in the morning before you eat.... If you need test strips or other testing supplies such as lancets let me know. I will plan to contact you via secure messaging in 4 weeks to discuss your blood sugar readings... - VA Pharmacist
Here, the pharmacist initiated a SM to inform the patient that the prescription was being mailed. The pharmacist also took this opportunity to inform the patient when to take the medication and inquired if the patient needed any other supplies.

\section{Patient Secure Message Response Content}

To further understand the potential impact of team-initiated SM on diabetes self-management and diabetes control, we examined the content of the SM sent by patients in response to team-initiated messages. A prominent theme was that teaminitiated messages served as gateways for patients to report on the status of diabetes-related outcomes and request help with their diabetes management. Twenty-nine percent $(n=59)$ of the responses asked to schedule an appointment. Twenty-two percent $(n=45)$ of responses pertained to prescription refills or renewals. In one example, a team-initiated SM asked about the patient's pain; the patient took advantage of this opportunity to report high blood sugar and request an appointment.

I'm still having pain in my lower back and upper legs. My sugar has been extremely high; 300 - 400. I really think I need to be checked for a UTI. Could you please make an appointment for me to come in to see the Dr.?

- VA patient

In another example, a RN asked a patient about their blood sugar readings. The patient took the opportunity to request

Table 3 Mediation Analysis Examining the Association of Team-Initiated SMs and Self-management Behavior and Self-efficacy, Mediated by Perceived Autonomy Supportive Health Care Climate (HCCQ score)

\begin{tabular}{|c|c|c|c|c|c|c|c|c|}
\hline \multirow[t]{2}{*}{ Outcome } & \multicolumn{4}{|c|}{ Diabetes self-management } & \multicolumn{4}{|c|}{ Diabetes self-efficacy } \\
\hline & $\beta$ & SE & $t$ & $p$ & $\beta$ & SE & $t$ & $p$ \\
\hline \multicolumn{9}{|c|}{ Model predicting perceived autonomy support } \\
\hline Age & 0.02 & 0.01 & 1.79 & 0.07 & 0.02 & 0.01 & 1.50 & 0.135 \\
\hline Gender & -0.22 & 0.43 & -0.50 & 0.616 & -0.32 & 0.41 & -0.77 & 0.443 \\
\hline Income & 0.52 & 0.18 & 2.83 & 0.005 & 0.50 & 0.18 & 2.72 & 0.007 \\
\hline Team-initiated secure messages & 0.43 & 0.21 & 2.01 & 0.046 & 0.39 & 0.21 & 1.85 & 0.065 \\
\hline \multicolumn{9}{|l|}{ Model predicting outcome variable } \\
\hline Age & 0.02 & 0.01 & 2.52 & 0.012 & 0.01 & 0.01 & 0.65 & 0.513 \\
\hline Gender & -0.50 & 0.20 & -2.43 & 0.016 & -0.73 & 0.42 & -1.74 & 0.083 \\
\hline Income & 0.01 & 0.01 & 1.33 & 0.184 & -0.16 & 0.19 & -0.87 & 0.387 \\
\hline Team-initiated secure messages* & -0.09 & 0.10 & -0.83 & 0.406 & 0.01 & 0.22 & 0.06 & 0.951 \\
\hline Perceived autonomy support & 0.09 & 0.02 & 3.66 & $<0.001$ & 0.25 & 0.05 & 4.92 & $<0.001$ \\
\hline \multirow[t]{2}{*}{ Indirect effect ${ }^{\dagger}$} & Indirect & $\mathrm{SE}$ & $95 \% \mathrm{CI}$ & & Indirect & SE & $95 \% \mathrm{CI}$ & \\
\hline & 0.04 & 0.02 & $0.003,0.082$ & & 0.10 & 0.05 & $-0.003,0.214$ & \\
\hline
\end{tabular}

*The direct effect of receiving at least one team-initiated SM on the outcome

tThe indirect effect of receiving at least one team-initiated SM on the outcome, as mediated by perceived autonomy support 
Table 4 Frequencies of Message Content

\begin{tabular}{|c|c|c|c|c|}
\hline \multirow[b]{2}{*}{ Message content } & \multicolumn{2}{|c|}{$\begin{array}{l}\text { Team- } \\
\text { initiated }\end{array}$} & \multicolumn{2}{|c|}{$\begin{array}{l}\text { Patient- } \\
\text { response }\end{array}$} \\
\hline & $n$ & $\%$ & $n$ & $\%$ \\
\hline Scheduling, referrals, or administrative & 83 & 36 & 59 & 29 \\
\hline Medication issue & 72 & 31 & 28 & 14 \\
\hline Medication renewal or refill & 39 & 17 & 45 & 22 \\
\hline Test result & 40 & 17 & 3 & 1 \\
\hline Test issue & 26 & 11 & 7 & 3 \\
\hline Self-reporting & 11 & 5 & 15 & 7 \\
\hline Health issue & 7 & 3 & 17 & 8 \\
\hline Technology & 6 & 3 & 4 & 2 \\
\hline Unknown/Other & 12 & 5 & 50 & 25 \\
\hline Personal connection & 1 & 0 & 0 & 0 \\
\hline Care coordination & 2 & 1 & 2 & 1 \\
\hline Informational & 3 & 1 & 9 & 4 \\
\hline Life issue & 0 & 0 & 5 & 2 \\
\hline Complaint & 0 & 0 & 2 & 1 \\
\hline
\end{tabular}

prescription refills: “...blood sugars are up and down I did try and adjust the insulin still working on it. I do need some refills omeprazole; morphine; hydrochlorothiazide; losartan; metformin; instant glucose; and melatonin... I will send you my numbers tomorrow..." The patient did send the requested blood sugar readings in a later message.

Team-initiated messages also resulted in productive diabetes management conversations. The following thread, which included 6 asynchronous SM exchanges, was initiated by a nurse on behalf of a physician concerned about the patient's $\mathrm{A} 1 \mathrm{C}$ level and recommended insulin titration.

Patient: "Wow that is terrible about the A1C; what is titration insulin?"

Nurse: "Titration of insulin means we would increase your Lantus based on your fasting blood sugar readings. Taking your... readings for 2 weeks would be helpful. We would review them and increase your insulin based on the readings..."

Patient: "The blood sugars were way up from Saturday [until] yesterday; in excess of $200 \ldots$ See all these ads for a lantus supplement that assists in lowering the A1C... Could that be an option?"

Nurse: "I don't think adding another oral med is going to work. Increasing insulin is the most effective way of getting blood sugars down. If you want to try diet changes then you can do that for a few weeks.... Your A1c is 8 not 18 you can more than likely get this down by cutting back on carbs; avoid frequent eating of foods really high in carbs and portion when you do allow it. I will put you down for a for a follow up contact in about 3 weeks..."

Patient: "...that sounds logical and will proceed with some oral discipline and add couple of units to my insulin. Thanks so much."

Patient (later): "My blood sugars have been ranging from 95 to 125. Still exercising 3-4 Times a week. Blood pressure never exceeding 140 over $70 . "$
From this team-initiated communication, the patient was able to learn about methods for better glucose management (e.g., insulin titration, diet change). The SM dialog was productive, with the patient reporting successful stabilization of blood glucose and engagement in physical activity.

\section{DISCUSSION}

Effective communication between patients and their clinical teams is critical for the continued care of managing a complex, chronic condition such as diabetes. SM through patient portals, such as My HealtheVet, offers teams the opportunity to easily communicate with their patients, which can in turn improve their disease management and health outcomes. Some healthcare systems have realized that clinical teams may be able to encourage SM use among their patients by initiating SM. For this reason, in the current study, we examined whether clinical teams could support diabetes selfmanagement and self-efficacy by initiating SM with their patients, and if autonomy support served as a mechanism within this relationship.

Patients who received at least one team-initiated SM were significantly more likely to perceive their healthcare climate as more autonomy-supportive compared to patients who did not receive any team-initiated communication. Patients that perceived a more autonomy-supportive healthcare climate were significantly more likely to report engaging in more diabetes self-management behaviors and more likely to report a higher sense of diabetes self-efficacy, which have been significantly related to better glucose control. ${ }^{16}$ The importance of a sense of autonomy for behavior change is a principle tenet in selfdetermination theory. ${ }^{8,}{ }^{9}$ To our knowledge, this is the first study to demonstrate that clinical team-initiated communication via SM can support patients' diabetes self-management behaviors by supporting their sense of autonomy.

There was an indirect effect between receiving at least one team-initiated SM and engaging in more diabetes selfmanagement behaviors via patients' perceived autonomy support. In other words, patients who were on a team that initiated at least one SM were more likely to engage in greater diabetes self-management due to a greater perception of an autonomysupportive healthcare climate. Previous qualitative work has pointed to ineffective patient-provider communication as one of most prominent barriers to diabetes self-management. ${ }^{24}$ In a previous trial, ${ }^{25}$ diabetic patients who were randomized to receive weekly automated telephone messages about their disease management were more engaged in their diabetes self-management compared to patients who were randomized to attend group medical visits. Such programs that offer more frequent communication appear extremely beneficial for promoting diabetes self-management. Our results extend this work and suggest that as little as a single SM has the potential to make a significant impact on patient autonomy and subsequent self-management. Team-initiated communication via 
SM that promotes a sense of autonomy can potentially open the door for patients to speak with their providers about other self-management issues. The patient SM responses that we analyzed offer compelling support for this assertion. Patients who received a team-initiated message were able to take advantage of the communication and engage in productive self-management behaviors, such as requesting appointments and prescription refills, and tracking diabetes-related outcomes like blood sugar readings. Patients also engaged in conversations with their team about behaviors that could help manage their diabetes, such as exercise and diet. These conversations may have helped patients learn more about these topics. Self-reported diabetes self-management and glycemic control is well-established, ${ }^{16}$ and it is promising that teaminitiated communication could potentially further contribute to greater diabetes self-management behaviors.

Though there was a significant relationship between perceived autonomy support and diabetes self-efficacy, there was no evidence for perceived autonomy support as a significant mediator between team-initiated SM and diabetes self-efficacy. Notably, there was a negative correlation between the number of team-initiated SMs and diabetes self-efficacy. There may be alternative explanations for this relationship. A recent literature review reported that patients with higher diabetes self-efficacy were more likely to be users of patient portals; ${ }^{26}$ teams may feel less compelled to initiate SM with patients who are more self-efficacious. Additionally, there may be moderating factors which were not assessed in this study. Previous reports between clinician factors and selfefficacy have pointed to clinician empathy as a moderator for patients' self-efficacy. Compared to clinicians with low empathy, patients with more empathetic clinicians were more likely to have a higher sense of self-efficacy. ${ }^{27}$

Registered nurses sent the majority of the team-initiated SMs. This aligns with prior work that identified registered nurses and licensed practical nurses as the most frequent senders or responders to $\mathrm{SM}^{23}$ Additionally, the majority of messages were transactional and focused on scheduling, referral, or administrative issues. A previous study found that patients valued the convenience and ease of communication provided by $\mathrm{SM},{ }^{28}$ suggesting that SM may provide an easily accessible substitution for potentially unnecessary in-person visits or telephone calls.

\section{Limitations and Future Directions}

Approximately $25 \%$ of the sample received a proactive, teaminitiated SM. This number is notably higher than previous reports of team-initiated messaging. ${ }^{23,}{ }^{29}$ In the current study, patients were recruited based on being sustained, current users of SM. Therefore, this sample is not necessarily representative of the average patient. Overall, survey respondents were older, less likely to have a mental health comorbidity, and used the patient portal more frequently. Within our survey sample, we observed a significant benefit from receiving a team-initiated message, but it is possible that proactive communication may not be as beneficial for other patients who use the portal less. Future studies would benefit from a controlled trial where patients are randomized to receive or not receive a proactive message from their healthcare team. Additionally, as is typical in Veteran samples, this sample was disproportionately male.

This was an observational study; though mediation analyses allow for the discussion of directionality and causality, we are limited in our interpretation of these relationships. Future work would benefit from examining these relationships from a longitudinal and/or interventional perspective to enable more conclusive discussions regarding causality. Using both quantitative and qualitative data sources, this study was able to examine the relationship between receiving a proactively initiated SM and patient-reported measures known to be associated with health outcomes, and was able to examine the content of the SM. Future lines of work may consider a more granular approach to examining the relationship between receiving certain types of messages from certain healthcare team members to evaluate if these differences impact a patients' sense of autonomy, diabetes self-management, and diabetes self-efficacy.

As of January 2020, over 3.3 million Veterans (roughly $52 \%$ of VA patients) were registered portal users, $32 \%$ of whom were active users of SM. As portal adoption continues to increase across the country, proactive, team-initiated messages have the potential to reach hundreds of thousands of patients, and subsequently support their disease self-management. In order to support this, future work must consider identifying optimal workflows to promote team-initiated SM.

\section{CONCLUSION}

This study demonstrated that patients who are assigned to clinical teams that initiate communication through SM are more likely to perceive a more autonomy-supportive healthcare climate. Patients who feel more autonomous regarding their healthcare are more likely to engage in more diabetes self-management behaviors and feel more self-efficacious managing their diabetes. Finally, teams that initiate communication may be able to indirectly enhance patient selfmanagement behaviors by promoting a sense of autonomy. Clinical teams should recognize the value of initiating autonomy-supportive communication with their patients and the potential of SM features that are part of many patient portals as a viable channel for doing so. This is particularly the case for complex chronic diseases such as diabetes that require ongoing, effective self-management between clinical visits.

Acknowledgments: The team would like to thank Carolyn Purington, Kathryn DeLaughter, and Linda Am for coding the secure messages. The contents of this manuscript do not represent the views of the Department of Veterans Affairs or the United States Government. 
Corresponding Author: Stephanie A. Robinson, PhD; Center for Healthcare Organization and Implementation Research Edith Nourse Rogers Memorial Veterans Hospital, Bedford, MA, USA (e-mail: Stephanie.Robinson5@va.gov).

Funding Information This research was financially supported by the Department of Veterans Affairs, Veterans Health Administration, Office of Research and Development, Health Services Research, and Development Service (IIR 15-307). The writing of this manuscript was financially supported by the Department of Veterans Affairs Office of Academic Affiliations Advanced Fellowship Program in Health Services Research and the National Heart, Lung, and Blood Institute (K12HL138049).

\section{Compliance with Ethical Standards:}

Conflict of Interest: The authors declare that they do not have a conflict of interest.

\section{REFERENCES}

1. Weppner WG, Ralston JD, Koepsell TD, et al. Use of a shared medical record with secure messaging by older patients with diabetes. Diabetes Care. 2010;33(11):2314-2319.

2. Ricciardi L, Mostashari F, Murphy J, Daniel JG, Siminerio EP. A national action plan to support consumer engagement via e-health. Health Affairs. 2013;32(2):376-384

3. Zhou YY, Kanter MH, Wang JJ, Garrido T. Improved quality at Kaiser Permanente through e-mail between physicians and patients. Health Affairs. 2010;29(7): 1370-1375

4. Harris LT, Koepsell TD, Haneuse SJ, Martin DP, Ralston JD. Glycemic control associated with secure patient-provider messaging within a shared electronic medical record: a longitudinal analysis. J Diabetes Care. 2013;36(9):2726-2733.

5. Shimada SL, Allison JJ, Rosen AK, Feng H, Houston TK. Sustained use of patient portal features and improvements in diabetes physiological measures. J Med Internet Res. 2016;18(7).

6. Lyles CR, Grothaus L, Reid RJ, Sarkar U, Ralston JD. Communication about diabetes risk factors during between-visit encounters. Am J Manag Care. 2012;18(12):807-815.

7. Wolcott V, Agarwal R, Nelson DA. Is Provider Secure Messaging Associated With Patient Messaging Behavior? Evidence From the US Army. J Med Internet Res. 2017;19(4):e103.

8. Deci EL, Ryan RM. Facilitating optimal motivation and psychological well-being across life's domains. Canadian Psychology/Psychologie canadienne. 2008;49(1):14

9. Ng JY, Ntoumanis N, Thøgersen-Ntoumani C, et al. Self-determination theory applied to health contexts: A meta-analysis. Perspect Psychol Sci. 2012;7(4):325-340.

10. Williams GC, Patrick H, Niemiec CP, et al. Reducing the health risks of diabetes. Diabetes Educ. 2009;35(3):484-492.

11. Williams GC, MCGregor HA, King D, Nelson CC, Glasgow RE. Variation in perceived competence, glycemic control, and patient satisfaction: relationship to autonomy support from physicians. Patient Educ Couns. 2005;57(1):39-45.

12. Koponen AM, Simonsen N, Laamanen R, Suominen $\mathbf{S}$. Health-care climate, perceived self-care competence, and glycemic control among patients with type 2 diabetes in primary care. Health Psychol Open. 2015;2(1):2055102915579778.

13. Williams GC, Lynch M, Glasgow RE. Computer-assisted intervention improves patient-centered diabetes care by increasing autonomy support. Health Psychol. 2007;26(6):728.

14. Su Y-L, Reeve J. A meta-analysis of the effectiveness of intervention programs designed to support autonomy. Educ Psychol Rev. 2011;23(1):159-188.

15. Williams GC, Rodin GC, Ryan RM, Grolnick WS, Deci EL. Autonomous regulation and long-term medication adherence in adult outpatients. Health Psych. 1998;17(3):269.

16. Schmitt A, Gahr A, Hermanns N, Kulzer B, Huber J, Haak T. The Diabetes Self-Management Questionnaire (DSMQ): development and evaluation of an instrument to assess diabetes self-care activities associated with glycaemic control. Health Qual Life Outcomes. 2013;11(1): 138 .

17. Lorig K, Ritter PL, Villa FJ, Armas J. Community-based peer-led diabetes self-management. Diabetes Educ. 2009;35(4):641-651.

18. Johnson B, Christensen L. Educational research: Quantitative, qualitative, and mixed approaches. Sage; 2008.

19. Hayes AF. Introduction to mediation, moderation, and conditional process analysis: A regression-based approach. Guilford Publications; 2017.

20. Kravitz RL, Bell RA, Franz CE. A Taxonomy of Requests by Patients (TORP) A New System for Understanding Clinical Negotiation in Office Practice. J Fam Pract. 1999;48(11):872-872.

21. Sittig DF. Results of a content analysis of electronic messages (email) sent between patients and their physicians. BMC Med Inform Decis Mak. 2003;3(1): 11 .

22. Miller EA, Nelson E-L. Modifying the Roter Interaction Analysis System to study provider-patient communication in telemedicine: promises, pitfalls, insights, and recommendations. Telemed J E Health. 2005; 11(1):44-55.

23. Shimada SL, Petrakis BA, Rothendler JA, et al. An analysis of patientprovider secure messaging at two Veterans Health Administration medical centers: message content and resolution through secure messaging. J Am Med Inform Assoc. 2017;24(5):942-949.

24. Matthews SM, Peden AR, Rowles GD. Patient-provider communication: Understanding diabetes management among adult females. Patient Educ Couns. 2009;76(1):31-37.

25. Schillinger $\mathbf{D}$, Hammer $\mathbf{H}$, Wang $\mathbf{F}$, et al. Seeing in 3-D: examining the reach of diabetes self-management support strategies in a public health care system. 2008;35(5):664-682.

26. Sun R, Korytkowski MT, Sereika SM, Saul MI, Li D, Burke LE. Patient portal use in diabetes management: Literature review. Jmir Diabetes. 2018;3(4):e11199.

27. Flickinger TE, Saha S, Roter $\mathbf{D}$, et al. Clinician empathy is associated with differences in patient-clinician communication behaviors and higher medication self-efficacy in HIV care. Patient Educ Couns. 2016;99(2):220226.

28. Haun JN, Lind JD, Shimada SL, et al. Evaluating user experiences of the secure messaging tool on the Veterans Affairs' patient portal system. J Med Internet Res. 2014;16(3):e75.

29. Hogan TP, Luger TM, Volkman JE, et al. Patient centeredness in electronic communication: evaluation of patient-to-health care team secure messaging. J Med Internet Res. 2018;20(3):e82.

Publisher's Note: Springer Nature remains neutral with regard to jurisdictional claims in published maps and institutional affiliations. 